\title{
THE RADIOCARBON INTRACAVITY OPTOGALVANIC SPECTROSCOPY SETUP AT UPPSALA
}

\author{
Gerriet Eilers $^{1} \cdot$ Anders Persson $^{1} \bullet$ Cecilia Gustavsson ${ }^{1} \cdot$ Linus Ryderfors $^{1} \bullet$ Emad Mukhtar $^{2} \bullet$ \\ Göran Possnert ${ }^{1}$ - Mehran Salehpour ${ }^{1,3}$
}

\begin{abstract}
Accelerator mass spectrometry (AMS) is by far the predominant technology deployed for radiocarbon tracer studies. Applications are widespread from archaeology to biological, environmental, and pharmaceutical sciences. In spite of its excellent performance, AMS is expensive and complicated to operate. Consequently, alternative detection techniques for ${ }^{14} \mathrm{C}$ are of great interest, with the vision of a compact, user-friendly, and inexpensive analytical method. Here, we report on the use of intracavity optogalvanic spectroscopy (ICOGS) for measurements of the ${ }^{14} \mathrm{C} /{ }^{12} \mathrm{C}$ ratio. This new detection technique was developed by Murnick et al. (2008). In the infrared (IR) region, $\mathrm{CO}_{2}$ molecules have strong absorption coefficients. The IR-absorption lines are narrow in line width and shifted for different carbon isotopes. These properties can potentially be exploited to detect ${ }^{14} \mathrm{CO}_{2},{ }^{13} \mathrm{CO}_{2}$, or ${ }^{12} \mathrm{CO}_{2}$ molecules unambiguously. In ICOGS, the sample is in the form of $\mathrm{CO}_{2}$ gas, eliminating the graphitization step that $\mathrm{h}$ is required in most AMS labs. The status of the ICOGS setup in Uppsala is presented. The system is operational but not yet fully developed. Data are presented for initial results that illustrate the dependence of the optogalvanic signal on various parameters, such as background and plasma-induced changes in the sample gas composition.
\end{abstract}

\section{INTRODUCTION}

Isotope ratio measurements provide valuable information in a number of pure and applied disciplines of science. Due to the extremely low abundance of ${ }^{14} \mathrm{C}$ in most samples, accelerator mass spectrometry (AMS) has been the main choice for such measurements, replacing the traditional decay counting techniques. AMS has been used to perform precise isotope ratio measurements with sensitivities in the $10^{15}: 1$ range for carbon, resulting in a number of applications in diverse disciplines including archaeology and environmental sciences. A growing segment in AMS is in the biomedical field where ${ }^{14} \mathrm{C}$-labeled molecules are used as a biological marker in, for example, pharmaceutical drugs. The biological applications have pushed the sensitivity limit of quantification down to $120 \mathrm{zmol}\left(1 \mathrm{zmol}=10^{-21} \mathrm{~mol}\right)$ in absolute sample amount (Salehpour et al. 2008). A noteworthy application of AMS in the pharmaceutical research and development sector is microdosing (Lappin and Garner 2003), which is based on administrating very small doses of drugs, below $100 \mu \mathrm{g}$, directly to humans. The increased interest in such new applications for AMS has prompted development of alternative techniques, partly because AMS has some major limitations, e.g. the high capital and running costs as well as the complexity of operating a particle accelerator. Furthermore, AMS suffers from limitations in the minimum sample size, which has implications for a number of applications with limited sample amounts. Any new technique must be able to provide a comparable sensitivity and sample size to that which is possible with AMS, in order to become an alternative in these new fields. Furthermore, a less destructive method than AMS is of greater interest.

New developments in this direction are based on laser spectroscopy technology, and have the potential to provide a low-cost and easy-to-operate table-top experimental setup. In recent years, 2 new laser-based techniques have been reported: intracavity optogalvanic spectroscopy (ICOGS) (Murnick et al. 2008) and cavity ring down (CRD) spectroscopy (Galli et al. 2011). Both these techniques are based on high-resolution, ultrasensitive infrared absorption spectroscopy. The sample is oxidized to $\mathrm{CO}_{2}$ and the isotopic species of the gas are distinguished from the shift in the infrared absorption spectra due to the difference in the mass of the carbon atoms (Labrie and Reid 1981).

\footnotetext{
${ }^{1}$ Dept. of Physics and Astronomy, Ion Physics, Box 516, SE-751 20, Uppsala, Sweden.

${ }^{2}$ Dept. of Chemistry, Ångström Laboratory, Box 523, SE-751 20, Uppsala, Sweden.

${ }^{3}$ Corresponding author: mehran.salehpour@physics.uu.se.
} 


\section{G Eilers et al.}

These techniques require the use of highly stable, single-frequency lasers. Unlike AMS, the laserbased measurement techniques do not destroy the $\mathrm{CO}_{2}$ sample, so that it can be stored and used in repetitive measurements.

ICOGS is based on the optogalvanic (OG) effect. The interaction of the incident laser with atoms or molecules present in a glow discharge (plasma) induces changes in the electrical properties (impedance) of the plasma, which can be measured electrically. The measured impedance change is proportional to the number of interacting molecules, but also will depend on the plasma parameters, e.g. pressure, as well as the laser intensity and gas composition in the discharge (Ilkmen 2009).

In ICOGS, the plasma cell containing the sample, here referred to as the sample cell, is placed inside the laser cavity (Murnick et al. 2008), rather than outside the laser as it is in the conventional absorption method (Murnick and Peer 1994). In the intracavity experiment, 2 stabilized, single-frequency lasers with the active lasing media being ${ }^{12} \mathrm{CO}_{2}$ and ${ }^{14} \mathrm{CO}_{2}$, respectively, were used to perturb the plasma in the sample cell. High sensitivity was reported down to isotope ratios in the $10^{-13}$ range. However, the technique suffers from problems with calibration over a wide ${ }^{14} \mathrm{CO}_{2}$ concentration range (Ilkmen 2009) as well as a large background.

Galli et al. (2011) have recently reported another technique in which saturated-absorption CRD spectroscopy is used to measure the ${ }^{14} \mathrm{C} /{ }^{12} \mathrm{C}$ ratio. In $\mathrm{CRD}$, the gas sample cell is placed between 2 highly reflective mirrors that form a Fabry-Perot cavity. The infrared light from a laser, tuned to a specific ro-vibrational transition of $\mathrm{CO}_{2}$, enters the cavity and will undergo multiple reflections between the cavity mirrors. The high reflectivity of the mirrors ensures that photons can make many round trips resulting in a long effective path, and consequently an ultrasensitive absorption measurement. The authors report a linear response in concentration down to 43 parts per quadrillion, i.e. $43: 10^{15}$ (Galli et al. 2011). However, this method requires a rather complicated laser system to obtain stable single-frequency operation at $4.5 \mu \mathrm{m}$. Furthermore, a relatively large sample size of about $70 \mathrm{mg}$ is required, which may limit its applications.

This article reports on the progress made in the development of an ultrasensitive ${ }^{14} \mathrm{C} /{ }^{12} \mathrm{C}$ detection system using ICOGS at Uppsala University. Details of the experimental setup are presented including methods of detection and control. We discuss the potentials and limitations of this technique, possible sources of the background in the experiment, and propose methods for improvement.

\section{METHODS}

\section{The Experimental Setup}

A schematic description of the experimental setup can be seen in Figure 1. The foundation of the setup is the $3 \mathrm{CO}_{2}$ lasers, with lasing frequencies stemming from the ro-vibrational transitions of ${ }^{12} \mathrm{C}^{16} \mathrm{O}_{2},{ }^{13} \mathrm{C}^{16} \mathrm{O}_{2}$, and ${ }^{14} \mathrm{C}^{16} \mathrm{O}_{2}$. Here, the ${ }^{12} \mathrm{C}^{16} \mathrm{O}_{2}$ laser was a model Merit-SL, and the ${ }^{13} \mathrm{C}^{16} \mathrm{O}_{2}$ laser was a model L20-SZ, both single longitudinal mode lasers from Access Laser Co. (Everett, WA, USA). The beams from the 3 lasers were power modulated using 3 optical choppers with orthogonal chopping frequencies (Figure 1a). This enabled separation of the OG signal, induced by the different lasers, in the frequency domain. The ${ }^{14} \mathrm{C}^{16} \mathrm{O}_{2}$ laser was a single longitudinal mode laser, custom made by LTG Lasertech Group Inc. (Concord, ON, Canada). The laser cavity of the ${ }^{14} \mathrm{CO}_{2}$ laser contained a grating on one end and an output coupler with piezo position control on the other end. The piezo positioning controlled the length of cavity, and consequently the laser wavelength (Figure 1a), with a maximum stroke of $L=7 \mu \mathrm{m}$. The laser had a maximum output power of $\sim 11 \mathrm{~W}$ at $11.8 \mu \mathrm{m}$. The length of the laser cavity was extended to $1.7 \mathrm{~m}$ to accommodate the intracavity sample cell and 


\section{${ }^{14}$ C Intracavity Optogalvanic Spectroscopy Setup at Uppsala}

an internal optical chopper. The output power was reduced by about $20 \%$ by the introduction of the sample cell, mostly due to losses in the Brewster windows, and by $55 \%$ by the chopper, due to the power modulation of the laser beam.
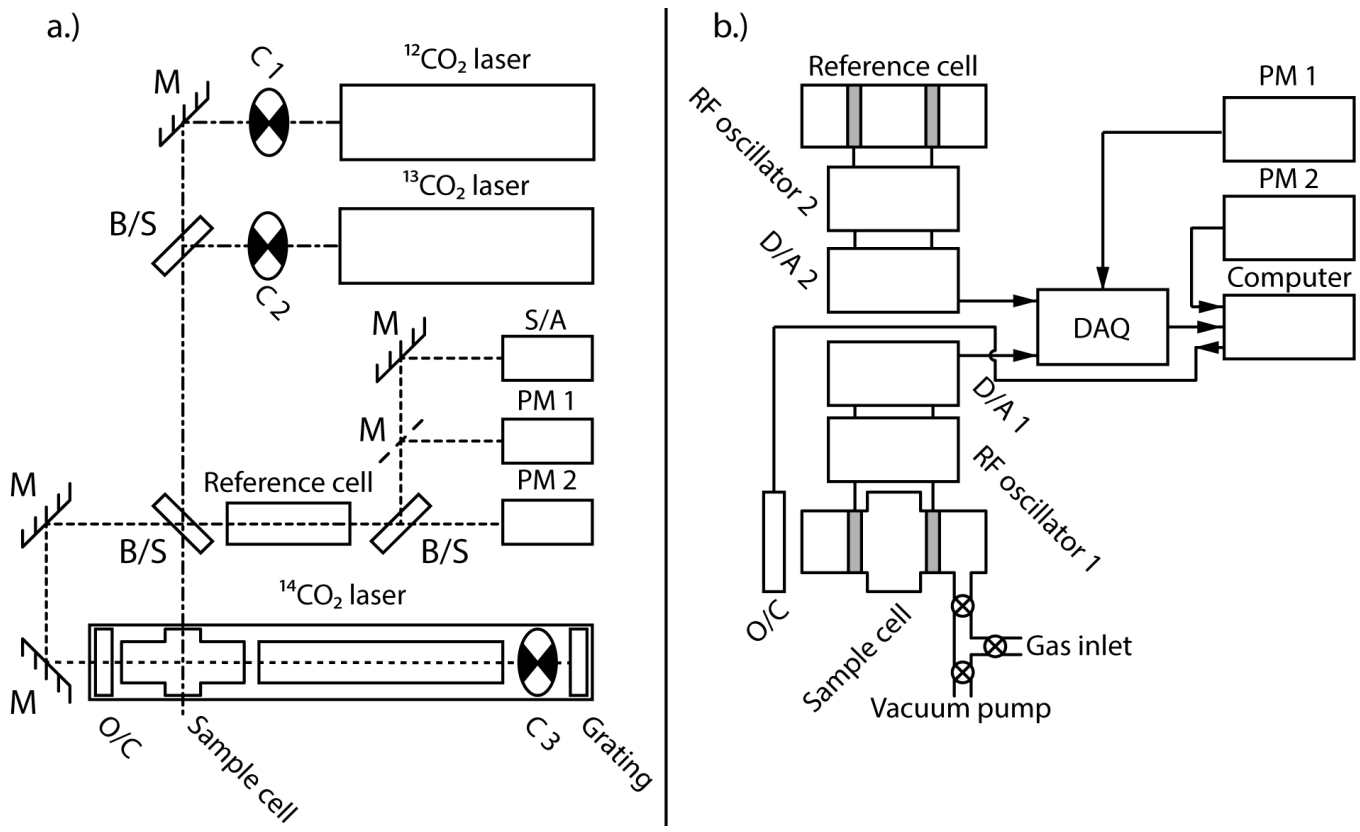

Figure 1 Schematic description of the optics (a) and electronics (b) of the experimental setup (abbreviations: $\mathrm{M}=$ steering mirror; $\mathrm{O} / \mathrm{C}=$ output coupler; $\mathrm{PM}=$ power meter; $\mathrm{B} / \mathrm{S}=$ beam splitter; $\mathrm{C}=$ chopper; $\mathrm{RF}=$ radio frequency; $\mathrm{S} / \mathrm{A}=$ spectrum analyzer; $\mathrm{DAQ}=$ data acquisition unit; and $\mathrm{D} / \mathrm{A}=$ differential amplifier).

The laser outputs were monitored in both a $\mathrm{CO}_{2}$ Laser Spectrum Analyzer from Macken Instruments Inc. (Santa Rosa, CA, USA), and in 2 power meters, either a conventional thermopile Vega Laser Power and Energy Meter from Ophir Optronics Solutions Ltd. (Jerusalem, Israel) or a discrete pyroelectric sensor Model QS-I-Test from GenTech-EO (Quebec, QC, Canada). Here, the Ophir power meter was used for measuring the laser output power in situ, whereas the GenTech power meter with a faster reaction time was used for e.g. timing signals.

The setup contained 2 plasma cells: 1) a reference cell containing $0.5 \%{ }^{14} \mathrm{CO}_{2}, 4.5 \%{ }^{12} \mathrm{CO}_{2}$, and $95 \%$ air at a total pressure of $467 \mathrm{~Pa}$; and 2) a sample cell, which could be filled with an arbitrary gas mix at pressures down to tens of $\mathrm{Pa}$ (Figure 1a). In the reference cell, air was used as a buffer gas to reduce the dissociation of $\mathrm{CO}_{2}$ into $\mathrm{CO}$ in the plasma. The sample cell had a cross-beam geometry (Figure 2), allowing for the ${ }^{12} \mathrm{CO}_{2}$ and the ${ }^{13} \mathrm{CO}_{2}$ laser beams to be passed through the cell transversely to the ${ }^{14} \mathrm{CO}_{2}$ beam (Figure 2). In the direction of the ${ }^{14} \mathrm{CO}_{2}$ beam, the cell was equipped with Brewster angle $\mathrm{ZnSe}$ windows to minimize the power loss in the laser. Plasma in each cell was excited by RF power coupled capacitatively into the cell from an RF oscillator operating at $10 \mathrm{MHz}$ or $40 \mathrm{MHz}$ and $\sim 5 \mathrm{~W}$ (Figure 2). In accordance with the design by Murnick et al. (2008), the RF oscillators consisted of a tunable oscillating circuit and a transistor (May and May 1986). The OG signal manifested itself as a change in the plasma impedance and could be quantified by measuring the amplitude of the RF wave in the oscillator. The OG signal was amplified by a custom-made differential amplifier, with a variable gain of $40 \mathrm{~dB}$, and sampled by a 4-channel BNC-2110 data acquisition (DAQ) unit from National Instruments Corp. (Austin, TX, USA). Apart from the OG signals from the sample and 


\section{G Eilers et al.}

reference cells, the output of the pyroelectric power meter was recorded by the DAQ, whereas the thermopile power meter was connected to the computer directly via a USB interface.

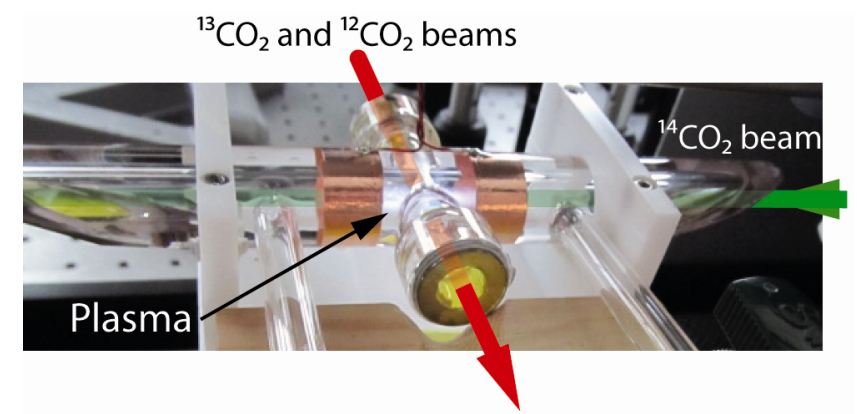

Figure 2 Photograph of the sample cell with an ignited plasma. The directions of the laser beams are indicated in the picture. Copper tape forms the electrodes for the capacitive coupled plasma.

The piezoelectric tuning of the cavity length of the ${ }^{14} \mathrm{CO}_{2}$ laser was controlled by the computer via a RS-232 interface. This control was based on the feedback from either one of the power meters or the reference cell OG signal. The ${ }^{14} \mathrm{CO}_{2}$ laser could be either scanned through its gain profile or locked at the maximum intensity of a particular laser line.

The inputs from the DAQ and the output to the piezo control were processed and controlled by custom software written in LabVIEW 2010 (10.0, National Instruments Inc., Austin, TX, USA). The DAQ had a sample rate of $1 \mathrm{kHz}$, and the software transformed series of 1000 time samples (Figure 3a), into the frequency domain using Fourier transformation, i.e. each transformation consisted of 1000 samples over a total of $1 \mathrm{~s}$. The OG signal was defined as the integral of the peak corresponding to the particular chopping frequency, in an interval given by a variable base parameter, typically set to about $10 \%$ of the peak amplitude (Figure $3 \mathrm{~b}$ ). Consequently, the sample rate of the OG signal was $1 \mathrm{~Hz}$. In the initial stages of the project, these parameters were varied to find the optimum settings. It was found that changing the sample and transformation rates did not have a strong influence on the signal-to-noise ratio, and neither did the base parameter, as long as it clearly exceeded the noise floor. The data in Figure 3 were measured with the sample cell and the ${ }^{12} \mathrm{CO}_{2}$ laser.

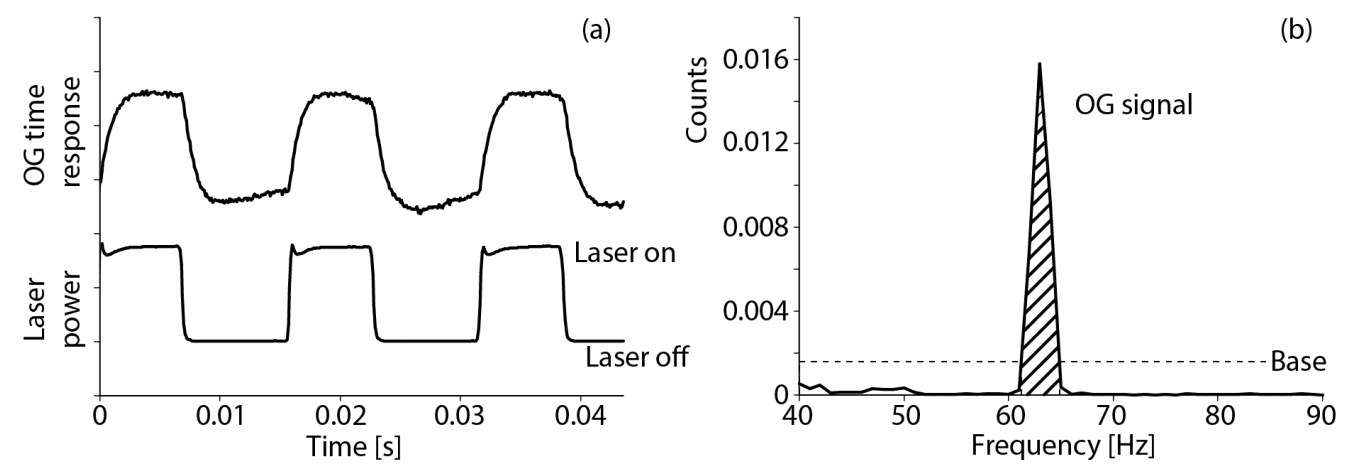

Figure 3 Typical time profile of the OG response of the plasma (a), with the upper curve showing the OG time response and the lower curve showing the power of the ${ }^{12} \mathrm{CO}_{2}$ laser. To the right is the Fourier transform of the OG time sequence (b), where the cross-hatched area under the peak indicates the integral corresponding to the OG signal. 


\section{${ }^{14}$ C Intracavity Optogalvanic Spectroscopy Setup at Uppsala}

\section{Measurement Modes}

There are 2 alternative approaches to the $\mathrm{CO}_{2}$ sample handling in ICOGS:

1. The static mode (Batch mode) where the gas is confined to a well-defined volume. The advantage of this method is twofold: a) The sample can be recovered after use; and b) the amount of sample needed is small, facilitating analysis of $\mu \mathrm{g}$ samples.

2. The dynamic mode (Flow mode), where the sample is continuously exchanged and renewed. The advantages of this method include continuity in plasma operation for better signal stability, and consequently better signal reproducibility.

In this study, we have worked solely with the static mode as the perceived advantages were deemed important for our application, namely small biological samples in the $\mu \mathrm{g}$ carbon range. Before analysis, the water content in the analyzed gases was removed in a $-80{ }^{\circ} \mathrm{C}$ cold trap as this has a detrimental effect on the reproducibility of the OG signal (D Murnick, private communication).

\section{MEASUREMENTS}

In the standard measurement procedure, a laser transition was first chosen by tuning of the grating of the ${ }^{14} \mathrm{CO}_{2}$ laser using micrometer screws. Secondly, the lasing frequency was locked by tuning of the cavity length to the center of the transition. This was done by using the piezo controller with feedback from the fast power meter or the reference cell. The cavity length was then stable over timescales up to $1 \mathrm{hr}$. The OG response of the sample and reference cells could be monitored and the OG signal measured.

Alternatively, the piezo-controlled output coupler was used to vary the lasing frequency around the center of a laser transition, to study the spectral shape of the laser gain profile and/or the OG response of the $\mathrm{CO}_{2}$ molecules in the reference and sample cells. The frequency was typically varied about $\pm 54 \mathrm{MHz}$, corresponding to a wavelength shift of about $\pm 25 \mathrm{pm}$ around the peak. The maximum scan width of the output coupler corresponded to a variation of the length of the laser cavity of about $\pm 33 \mathrm{pm}( \pm 72 \mathrm{MHz})$. A measurement series consisted of a number of sweeps going from maximum to minimum frequency in steps of $0.9 \mathrm{MHz}$. At each step, the amplified signal from the RF oscillator was sampled by the DAQ for $1 \mathrm{~s}$. The sampled time sequence was then transformed into the frequency domain and the OG signal was calculated as described above. To improve the signalto-noise ratio, the final result was taken as the mean of multiple sweeps. In this paper, only results using the ${ }^{12} \mathrm{CO}_{2}$ and ${ }^{14} \mathrm{CO}_{2}$ lasers are presented.

\section{Residual Gas Analysis}

Apart from the OG measurements, a residual gas analyzer (RGA; Model XT100 from Extorr Inc., New Kensington, PA, USA) was used for analyzing the gas chemistry of the $\mathrm{CO}_{2}$ plasma. The RGA was a compact quadrupole mass spectrometer with integrated Pirani and ion gauges. It consisted of a probe (173 $\mathrm{mm}$ length, $35 \mathrm{~mm}$ diameter) that was inserted into the vacuum system. Attached to the probe was a communications and control unit (CCU) that contained the electronics for the probe control and computer communications. The device came with its own software for mass spectrum analysis and had a resolution of $<1 \mathrm{amu}$. The RGA experiments were performed offline in a separate setup. Here, a second sample cell, identical to the one used in the ICOGS measurements, was connected to the vacuum system where the RGA probe was inserted. In order to connect the sample cell, which operated $\sim 100 \mathrm{~Pa}$, to the RGA, which operated at pressures below $10^{-4} \mathrm{~Pa}$, a fused silica capillary column from Supleco Inc. (Bellefonte, PA, USA), intended for use in gas chromatography, was used. The capillary column provided a continuous and very small gas flow between the 2 parts 


\section{G Eilers et al.}

of the system. The inner diameter of the column was $0.32 \mathrm{~mm}$ and the length of the column was $40 \mathrm{~cm}$. The RGA was pumped by a turbopump $(65 \mathrm{~L} / \mathrm{s})$, backed by a membrane vacuum pump, where the latter also was used to evacuate the sample cell. The timescale of the gas transfer from the sample cell to the RGA was less than $1 \mathrm{~s}$.

\section{Simulations}

An important concept in order to understand the response of a $\mathrm{CO}_{2}$ plasma to resonant irradiation is the spectral line shape of the transitions between different ro-vibrational states in general, and the broadening of the line profile, due to e.g. collisions in the gas, in particular. ICOGS of $\mathrm{CO}_{2}$ utilizes the transition between rotational quantum levels in the first excited vibrational level of the asymmetric stretch, denoted $\left(00^{0} 1\right)$, and corresponding rotational quantum levels in the first excited vibrational level of the symmetric stretch mode, denoted $\left(10^{\circ} 0\right)$, in the molecules, i.e. the same transitions that are utilized in an $\mathrm{CO}_{2}$ laser. For these transitions, the selection rule $\Delta J= \pm 1$ applies. Here, $J$ is the rotational quantum number. The transitions can be divided into 2 branches ( $\mathrm{R}$ and $\mathrm{P}$ ) where a transition from level $J$ to $J+1$ corresponds to the $\mathrm{P}$ branch and a transition from $J$ to $J-1$ corresponds to the $\mathrm{R}$ branch.

In an ideal case, the spectral lines are distinct and well separated, but in reality, 2 effects in particular will cause broadening of the lines - namely, collision and Doppler broadening (Silfvast 1996). Collision broadening is caused by the reduction of the lifetime of the excited states due to collisions between the molecules. The collision broadening of the spectral line, $S_{C}$, at frequency $f$, is homogeneous around the center frequency $f_{0}$, and can be described by a Lorentzian distribution (Demtröder 1981):

$$
S_{C}(f)=\frac{\alpha_{L}}{\pi} \cdot \frac{1}{\left(f-f_{0}\right)^{2}+\alpha_{L}^{2}}
$$

where $\alpha_{L}$ is the half width at half maximum of the broadening and is proportional to the collision rate. Assuming ideal gas conditions for a plasma consisting of pure $\mathrm{CO}_{2}, \alpha_{L}$ is given by (Silfvast 1996):

$$
\alpha_{L}=\frac{p \sigma}{2 \pi k_{B} T} \sqrt{\frac{8 k_{B} T}{\pi m}}
$$

where $p$ is the pressure, $T$ is the temperature, $m$ is the mass of the $\mathrm{CO}_{2}$ molecule, $k_{B}$ is Boltzmann's constant, and $\sigma$ is the collision cross-section between $2 \mathrm{CO}_{2}$ molecules. Here, the temperature was estimated as $T=340 \mathrm{~K}$ from measurements on a similar plasma (Deju et al. 1968) and the collision cross-section to $\sigma=1.57 \times 10^{-18} \mathrm{~m}^{2}$ (Meyer et al. 1993). For a plasma with several species, interspecies collisions have to be accounted for, making the equations somewhat more complicated (Ilkmen 2009).

The Doppler broadening stems from the distribution of velocities of the molecules in the inertial system of the system (Silfvast 1996). For each velocity, this will cause a Doppler shift, in turn causing an inhomogeneous broadening, $\mathrm{S}_{D}(f)$, of the spectral line peak around $f_{0}$, which can be described by a Gaussian distribution (Ilkmen 2009): 
${ }^{14}$ C Intracavity Optogalvanic Spectroscopy Setup at Uppsala

$$
S_{D}(f)=\frac{1}{\alpha_{G}} \cdot \sqrt{\frac{4 \ln (2)}{\pi}}\left[-4 \ln (2) \cdot \frac{\left(f-f_{0}\right)^{2}}{\alpha_{G}^{2}}\right]
$$

where $\alpha_{G}$ is the full width at half maximum of the broadening given by

$$
\alpha_{G}=\frac{2 f_{0}}{c} \sqrt{\frac{2 \ln (2) k_{B} T}{m}}
$$

and $c$ is the speed of light. The actual line profile will be given by a combination of the 2 effects known as the Voigt profile. Mathematically, the Voigt profile, $S_{V}$, is given by the convolution of the Gaussian and Lorentzian distributions, which is given by the integral (Huang and Yung 2004):

$$
S_{V}\left(f-f_{0}\right)=\int_{-\infty}^{\infty} S_{D}\left(f^{\prime}-f_{0}\right) S_{C}\left(f-f^{\prime}\right) d f^{\prime}
$$

which can be evaluated as the real part of the complex error function $w(z)$ given by:

$$
S_{V}(f)=\frac{1}{\alpha_{G}} \cdot \sqrt{\frac{4 \ln (2)}{\pi}} \cdot \operatorname{Re}\left[w\left(\frac{4 \ln (2)}{\alpha_{G}} \cdot\left[\left(f-f_{0}\right)+i \alpha L\right]\right)\right]
$$

A major concern in ICOGS is distinguishing the ${ }^{14} \mathrm{CO}_{2}$ signal from the background. The background primarily stems from the tail of the collision broadened peaks of ${ }^{12} \mathrm{CO}_{2}$ and ${ }^{13} \mathrm{CO}_{2}$ lines. It has been claimed that when estimating the background, it is sufficient to include only a few peaks in the closest vicinity of the ${ }^{14} \mathrm{CO}_{2}$ line that is being studied (Murnick et al. 2008; Ilkmen 2009). In order to investigate this further, a Matlab (R2010a) script simulating the spectrum of all the peaks of the transitions $\mathrm{P}(2)-\mathrm{P}(100)$ and $\mathrm{R}(0)-\mathrm{R}(58)$ for ${ }^{12} \mathrm{C}^{16} \mathrm{O}_{2},{ }^{13} \mathrm{C}^{16} \mathrm{O}_{2}$, and ${ }^{14} \mathrm{C}^{16} \mathrm{O}_{2}$ between $10-13 \mu \mathrm{m}$ was constructed. In order to do so, the peaks had to be scaled, firstly due to the population densities at different energy levels, and secondly due to the relative abundance of ${ }^{12} \mathrm{C},{ }^{13} \mathrm{C}$, and ${ }^{14} \mathrm{C}$. The population density of the different rotational levels, $N$, was assumed to follow a Boltzmann distribution:

$$
N(J) \alpha(2 J+1) \exp \left[-\frac{h c B J(J+1)}{k_{B} T}\right]
$$

where $h$ is Planck's constant and $B=39 \mathrm{~m}^{-1}$ is the rotational constant of $\mathrm{CO}_{2}($ Chedin 1975). Tabulated data (Bradley et al. 1986) was used in order to translate the ro-vibration transitions $\mathrm{P}(2)-\mathrm{P}(60)$ and $\mathrm{R}(0)-\mathrm{R}(58)$ into wavelengths. For the transitions $\mathrm{P}(62)-\mathrm{P}(100)$, there were only tabulated data for a few lines (Ilkmen 2009). Instead, the positions of $\mathrm{P}(62)-\mathrm{P}(100)$ were extrapolated from the data for $\mathrm{P}(2)-\mathrm{P}(60)$ using a quadratic polynomial fit. This approach caused a shift of the higher $\mathrm{P}$ lines of about $2 \mathrm{~nm}$. However, this shift was deemed insignificant for the investigations of this study, since the tail of the Lorentzian is not wavelength sensitive.

The relative abundance of the different carbon isotopes, along with the pressure and temperature, could be set arbitrarily in the script, making it a powerful tool in investigating the background over the whole spectrum or at specific P lines, for different samples and pressures. 


\section{G Eilers et al.}

\section{RESULTS AND DISCUSSION}

One of the key features of the ICOGS method is the unambiguous detection of ${ }^{14} \mathrm{C},{ }^{13} \mathrm{C}$, and ${ }^{12} \mathrm{C}$. As the sensitivity range for ${ }^{14} \mathrm{C} /{ }^{12} \mathrm{C}$ ratios is expected to be as low as $10^{-15}$ and extend orders of magnitude to higher isotopic ratios, a number of issues become important. These include various sources of noise and background, the linearity and dynamic range of the OG signal, as well as reproducibility. Here, we present the data obtained so far, focusing on some of these issues.

\section{Spectral Absorption}

In order to achieve the extreme sensitivity required for unambiguous detection of ${ }^{14} \mathrm{C}$, it is crucial to optimize the signal-to-background ratio of the OG signal - the background stemming from other carbon isotopes as well as other chemical species in the plasma. This can only be accomplished by a spectroscopic system with a maximum selectivity in terms of which ro-vibrational transitions that are utilized in the detection, since different transitions will have different signal-to-background ratios depending on their position in the spectrum (Ilkmen 2009). Ideally, only the transition with the best signal-to-background ratio should be studied, using a laser with a line width comparable to the absorption width of the transition. Consequently, it is imperative first to use a single-frequency laser with $\Delta \lambda / \lambda$ below $10^{-5}$, and second, that the detected species should have spectral distribution corresponding to that of the laser line. The first study aimed at demonstrating these issues. Figure 4 shows the laser power and OG signal measured as a function of the piezoelectric actuator's applied voltage (proportional to change in wavelength) for (a) ${ }^{12} \mathrm{C}^{16} \mathrm{O}_{2}$ and (b) ${ }^{14} \mathrm{C}^{16} \mathrm{O}_{2}$. The total scan range corresponds to $165 \mathrm{MHz}(63 \mathrm{pm})$ at $\lambda=10.6 \mu \mathrm{m}$ for ${ }^{12} \mathrm{C}^{16} \mathrm{O}_{2}$ and $108 \mathrm{MHz}(50 \mathrm{pm})$ at $\lambda=11.8 \mu \mathrm{m}$ for ${ }^{14} \mathrm{C}^{16} \mathrm{O}_{2}$. The data shows that the $\mathrm{OG}$ signal has a maximum that coincides with the laser power maximum for both isotopes. Since the laser power varies during the wavelength scan, the OG signal needs to be normalized (scaled) in order to get the correct absorption intensity, by dividing the OG signal with the measured laser power from one of the power meters or from the reference cell. This is shown for ${ }^{14} \mathrm{C}^{16} \mathrm{O}_{2}$ in Figure $4 \mathrm{~b}$. As will be presented in the Simulations section following, background sources have non-symmetric or flat spectral shapes, depending on the source of the background. In contrast, an OG signal closely resembling the laser line profile is strong evidence for a true ${ }^{14} \mathrm{C}$ signal. Equivalent measurements to Figure $4 \mathrm{a}$ and $\mathrm{b}$, with the sample cell filled with pure nitrogen, shows a flat OG signal that is independent of wavelength.

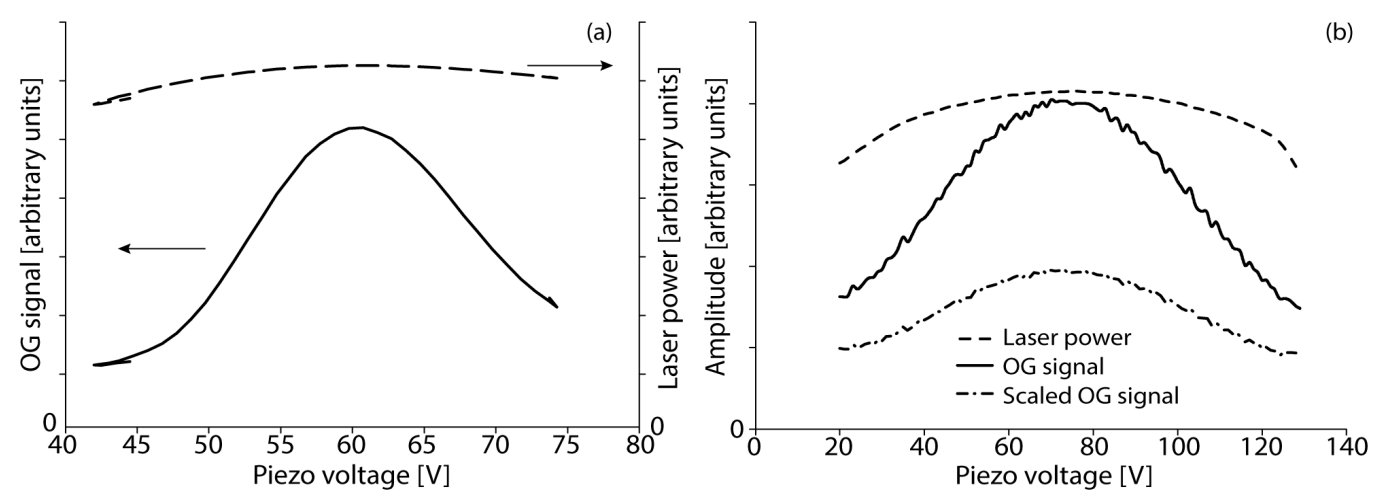

Figure 4 Spectral shape of the $\mathrm{P}(20)$ line of ${ }^{12} \mathrm{C}^{16} \mathrm{O}_{2}$ (a) measured in the sample cell at $\mathrm{p}=80 \mathrm{~Pa}$ with a mix of $10 \% \mathrm{CO}_{2}$ in $90 \% \mathrm{~N}_{2}$. The OG signal (solid line) was measured during a single piezo scan. The laser power (dashed line) is shown as a reference. Similarly, the spectral shape of the $\mathrm{P}(20)$ line of ${ }^{14} \mathrm{C}^{16} \mathrm{O}_{2}(\mathrm{~b})$ was measured in the reference cell at $\mathrm{p}=$ $467 \mathrm{~Pa}$ with a mix of $5 \% \mathrm{CO}_{2}$ in $95 \%$ air and a ${ }^{14} \mathrm{C} /{ }^{12} \mathrm{C}$ ratio of $\sim 10 \%$. Here, the curves represent an average over 57 piezo scans. The dash-dotted line shows the OG signal scaled by the laser power. 


\section{${ }^{14}$ C Intracavity Optogalvanic Spectroscopy Setup at Uppsala}

In order to unambiguously verify an OG signal at the transitions of interest (in this study $\mathrm{P}(20)$ and $\mathrm{P}(22)$ ), it can be assumed that the spectral shape of the OG signal should relate to the expected width and shape of the transition at a given pressure and temperature, i.e. the OG signal should reflect the absorption properties of $\mathrm{CO}_{2}$ in the sample cell. The homogeneous broadening caused by collisions and inhomogeneous broadening due to the Doppler shift have been calculated with the resultant Voigt distribution (Equation 6). Figure 5 shows the calculated Voigt profile and the measured OG signal as a function of the wavelength for $\mathrm{P}(20)$ of ${ }^{14} \mathrm{C}^{16} \mathrm{O}_{2}$ in the reference cell. As can be seen, the OG signal follows the expected Voigt profile with a coefficient of determination of $R^{2}=0.982$. The small deviation at the longer wavelengths is suspected to stem from background resulting from broadened nearby peaks.

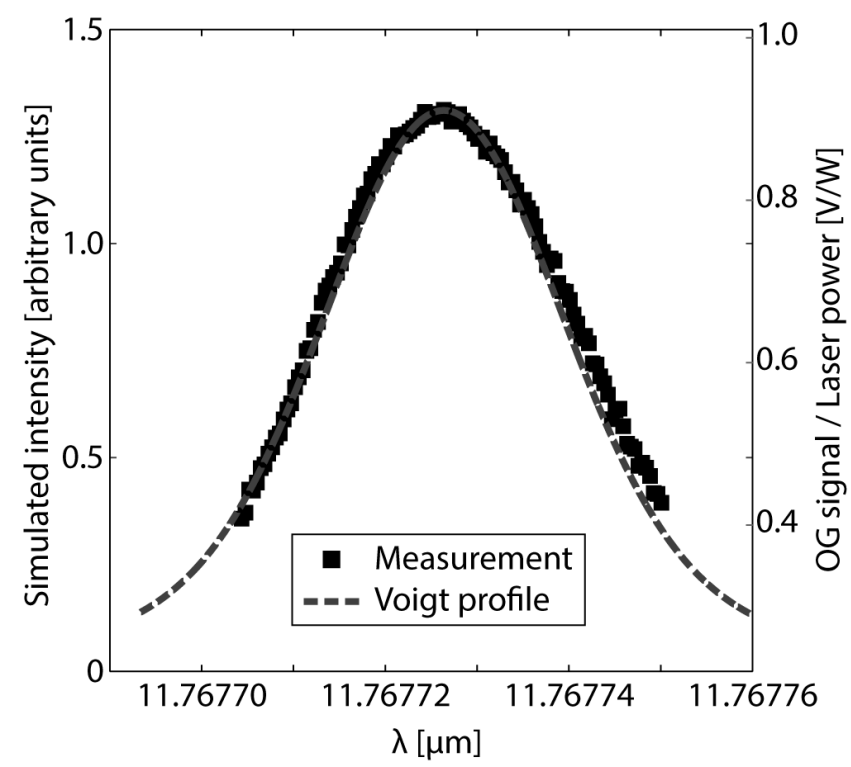

Figure 5 Simulated Voigt profile of the $\mathrm{P}(20)$ line of ${ }^{14} \mathrm{C}^{16} \mathrm{O}_{2}$ in the reference cell (dashed line) compared to the OG measurements (filled squares). The coefficient of determination of the fit was $R^{2}=0.982$.

The full width at half maximum (FWHM) of the Voigt profile was $67 \mathrm{MHz}$, corresponding to $\Delta \lambda / \lambda=$ $2.7 \times 10^{-6}$. This could be compared to the FWHM of the laser at $\mathrm{P}(20)$, which was specified to $144 \mathrm{MHz}$ or $\Delta \lambda / \lambda=5.7 \times 10^{-6}$. The main reason for the laser profile being broader than the OG response is the higher pressure in the laser tube $(2.7 \mathrm{kPa})$, which caused increased collisional broadening.

It should be noted that the data in Figure 5 were measured with the reference cell filled with a ${ }^{14} \mathrm{C}$ enriched gas corresponding to a ${ }^{14} \mathrm{CO}_{2} /{ }^{12} \mathrm{CO}_{2}$ ratio of $10 \%\left(\sim 8.5 \times 10^{10}\right.$ Modern). In order to study the sensitivity of the ICOGS setup and the effect of the intracavity enhancement factor, the OG response of the sample cell, filled with $5 \%$ of either 0.2 Modern or 10 Modern $\mathrm{CO}_{2}$ in $95 \% \mathrm{~N}_{2}$, was investigated. Here, $100 \mathrm{~Pa} \mathrm{CO}_{2}$ was mixed with $1900 \mathrm{~Pa} \mathrm{~N}_{2}$ after any residual water had been removed in a $-80{ }^{\circ} \mathrm{C}$ cold trap. The gas mix was then pumped to $100 \mathrm{~Pa}$ before the plasma was ignited and the measurement started. The standard measurement procedure was employed, where the ${ }^{14} \mathrm{CO}_{2}$ laser was tuned and locked to the center of the $\mathrm{P}(20)$ transition using feedback from the reference cell. The measurement was repeated 12 times where 2 measurements with 10 Modern $\mathrm{CO}_{2}$ 


\section{G Eilers et al.}

were followed by 2 measurements with 0.2 Modern $\mathrm{CO}_{2}$ and so forth. This was done to be able to distinguish any memory effects or long-time drift in the setup. Between the measurements, the system was pumped to $\sim 1 \mathrm{~Pa}$ after being purged with pure $\mathrm{N}_{2}$. The OG signal varied up to $\pm 10 \%$ between measurements on samples with the same ${ }^{14} \mathrm{C}$ content, but, more importantly, the signal differed insignificantly between samples with different ${ }^{14} \mathrm{C}$ content. In fact, the mean difference between the 0.2 Modern and the 10 Modern samples was only about half the standard deviation in the measurements, meaning that no clear dependence of the OG signal on the ${ }^{14} \mathrm{C}$ content could be distinguished.

Similar measurements to those of Figure 5 were performed with the sample cell at slightly lower pressure and with much lower ${ }^{14} \mathrm{CO}_{2} /{ }^{12} \mathrm{CO}_{2}$ ratios. Here, non-symmetric distributions were observed (Figure 6). As can be seen, the spectral shape of the laser power and the OG signal did not coincide, in this case for a sample with an activity of 10 Modern. The 2 distributions had dissimilar shapes and centroids, indicating a significant background contribution from non- ${ }^{14} \mathrm{CO}_{2}$ sources. This can also be seen from the shape of the scaled OG signal (Figure 6), which had a continuously increasing dependence on the piezo voltage, and consequently on the wavelength. Such a dependence can be interpreted as background from a nearby ${ }^{13} \mathrm{CO}_{2}$ or ${ }^{14} \mathrm{CO}_{2}$ line, in this case probably the $\mathrm{P}(68)$ line of ${ }^{13} \mathrm{C}^{16} \mathrm{O}_{2}$. Alternatively, a superposition of different distributions may give rise to similar observations. Consequently, an understanding of the background contribution from non- ${ }^{14} \mathrm{CO}_{2}$ sources is crucial.

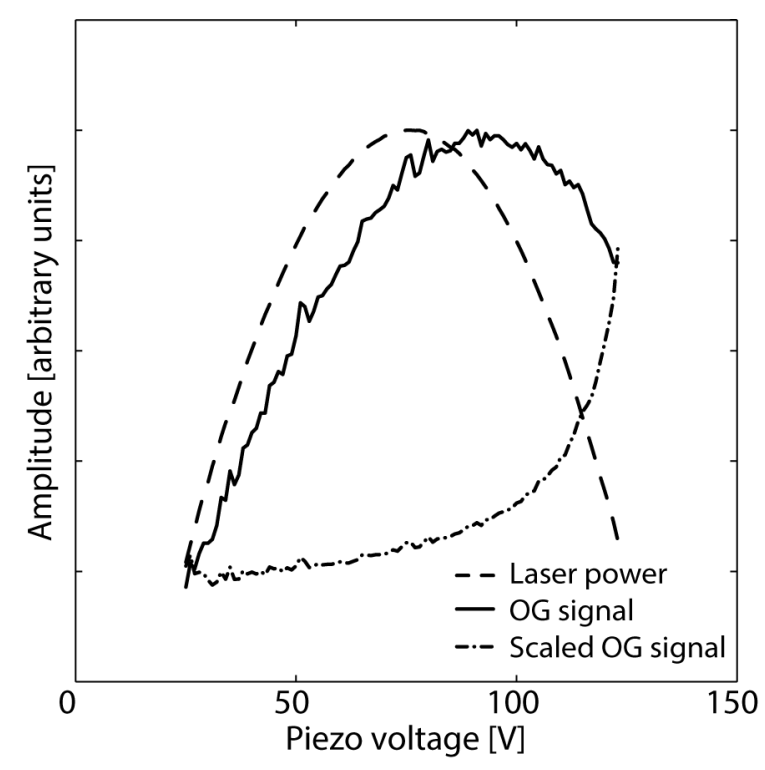

Figure 6 Spectral shape of the $\mathrm{P}(20)$ line of ${ }^{14} \mathrm{C}^{16} \mathrm{O}_{2}$ measured in the sample cell at $\mathrm{p}=40 \mathrm{~Pa}$ with a mix of $5 \% \mathrm{CO}_{2}$ in $95 \% \mathrm{~N}_{2}$. The 10 Modern OG signal (black solid line) was calculated from the mean of 166 piezo scans. The laser power (dashed line) and the scaled OG signal (dash-dotted line) are shown as a reference.

\section{Simulations}

The background contributions from ${ }^{12} \mathrm{CO}_{2}$ and ${ }^{13} \mathrm{CO}_{2}$ are of particular interest as they have transitions in the same spectral region as ${ }^{14} \mathrm{CO}_{2}$. In a first approximation, we ignore other oxygen isotopes 


\section{${ }^{14}$ C Intracavity Optogalvanic Spectroscopy Setup at Uppsala}

except ${ }^{16} \mathrm{O}$ due to lower abundance $\left(0.2 \%\right.$ for $\left.{ }^{18} \mathrm{O}\right)$. Figure 7 shows the calculated spectral distributions from the transitions $\mathrm{P}(20), \mathrm{P}(40), \mathrm{P}(60), \mathrm{P}(80)$, and $\mathrm{P}(100)$ for ${ }^{12} \mathrm{C}^{16} \mathrm{O}_{2}$ at $100 \mathrm{~Pa}$ over a broad spectrum. The $\mathrm{P}(20)$ line for ${ }^{14} \mathrm{CO}_{2}$, which is one of the lines of interest to this study, is shown for comparison. Here, the peaks are represented by the Lorentzian distribution (Equation 1), since the integral defining the complex error function, which is used to calculate the Voigt profile (Equation 6), cannot be evaluated in closed form. Instead, the integral is evaluated from its Taylor series, making it valid only relatively close to the peak. However, the Voigt profile approaches a Lorentzian function as $\left(f-f_{0}\right)$ increases whereby the latter can be regarded as a fair approximation far away from the peak (Huang and Yung 204), as is the case for the background in the spectrum relevant for ${ }^{14} \mathrm{C}$ ICOGS. Still, this approximation should only be regarded as a minimum transition probability far away from resonance, since e.g. transitions from non-electric dipole transitions will cause additional broadening and thus higher background. As can be seen, the background contribution from other ${ }^{12} \mathrm{CO}_{2}$ lines are significant, overshadowing the ${ }^{14} \mathrm{CO}_{2} \mathrm{P}(20)$ line. This contradicts the claim that only the background of the ${ }^{12} \mathrm{CO}_{2}$ lines closest to the ${ }^{14} \mathrm{CO}_{2}$ line measured has to be considered (Murnick et al. 2008; Ilkmen 2009), which is true only if the lines almost coincide. However, for the peaks investigated in this study, such a coincidence was only found for the $\mathrm{P}(2)$ line of ${ }^{14} \mathrm{C}^{16} \mathrm{O}_{2}$, which was situated merely $40 \mathrm{pm}$ away from the $\mathrm{P}(92)$ line of ${ }^{12} \mathrm{C}^{16} \mathrm{O}_{2}$. In the case of the ${ }^{14} \mathrm{C}^{16} \mathrm{O}_{2} \mathrm{P}(20)$ line, which is separated from the nearest ${ }^{12} \mathrm{CO}_{2}$ or ${ }^{13} \mathrm{CO}_{2}$ peak by more than 500 line widths (Murnick et al. 2008), the contribution from the densely populated ${ }^{12} \mathrm{CO}_{2}$ levels around $10.6 \mu \mathrm{m}$, and not the nearby $\mathrm{P}(100)$ peak, will dominate the background. This can be explained by the fact that the population density of the states with higher $J$ decays much faster than the tail of the Lorentzian. This can be seen from the dependency of the Lorentzian on the frequency shift away from $f_{0}$, which is $S_{C} \sim\left(f-f_{0}\right)^{-2} \sim\left(\lambda-\lambda_{0}\right)^{-2}$ (Equation 1), whereas for the Boltzmann distribution $N \sim$ $J \exp \left(-J^{2}\right)$, which could be interpreted as $N \sim\left(\lambda-\lambda_{0}\right) \exp \left(-\left(\lambda-\lambda_{0}\right)^{2}\right)$ (Equation 7). Hence, it is important to sum up the contribution of many ${ }^{12} \mathrm{CO}_{2}$ and ${ }^{13} \mathrm{CO}_{2}$ peaks in order to estimate the background in ${ }^{14} \mathrm{C}$ ICOGS, and not merely the ones closest to the ${ }^{14} \mathrm{CO}_{2}$ line that is being investigated. In this study, only the contributions from $\mathrm{P}(2)-\mathrm{P}(100)$ and $\mathrm{R}(0)-\mathrm{R}(58)$ for the $\left(00^{0} 1\right)-\left(10^{0} 0\right)$ band in ${ }^{12} \mathrm{C}^{16} \mathrm{O}_{2}$ and ${ }^{13} \mathrm{C}^{16} \mathrm{O}_{2}$ were included, but in a more thorough investigation, the contribution of different oxygen isotopes (Bradley et al. 1986), e.g. ${ }^{12} \mathrm{C}^{18} \mathrm{O}_{2}$, as well as the $\left(01^{1} 1\right)-\left(11^{1} 0\right)$ band with transitions around $11.0 \mu \mathrm{m}$ for ${ }^{12} \mathrm{C}^{16} \mathrm{O}_{2}$ (Hartmann and Kleman 1966), should be included. Moreover, transitions of other chemical species in the plasma such as $\mathrm{CO}, \mathrm{O}_{2}$, and $\mathrm{N}_{2}$ should be investigated.

Nevertheless, the calculations provide a reliable minimum possible background. Furthermore, the method is accurate enough to make the conclusion that for pure $\mathrm{CO}_{2}$ samples with isotopic ratio around 1 Modern, the signal is expected to be dominated by the background from ${ }^{13,12} \mathrm{CO}_{2}$ transitions, and requires extremely high stability for proper background subtraction.

\section{Signal Stability and Reproducibility}

One important parameter that is of central importance for analytical measurements is the accuracy and precision of the method, requiring well-defined reproducibility and stability over the analysis time. We encountered significant instabilities in the OG signal even though stable operation was ensured for the laser's power and wavelength, as well as for the plasma's electrical properties. This can be seen from the variation of the OG signal over time at 5 different pressures (Figure 8a). These variations led to questions regarding the effect of plasma-induced changes in gas chemistry. Traditionally, it would be expected that the concentration of the various radicals and other species in the plasma change in a monotone way with respect to time and continuously evolve towards equilibrium. However, our findings indicate the existence of more complex mechanisms. One important reaction that occurs in the sample cell after plasma ignition is the decomposition of $\mathrm{CO}_{2}$ into $\mathrm{CO}$ and 


\section{G Eilers et al.}

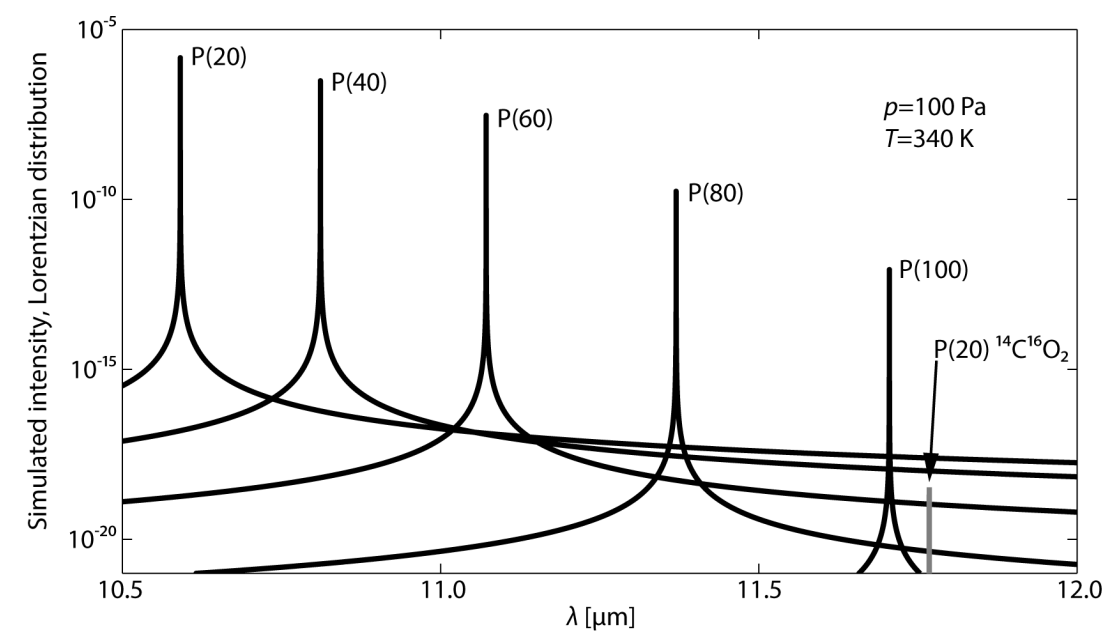

Figure 7 Simulated background from ${ }^{12} \mathrm{C}^{16} \mathrm{O}_{2}$ transitions $\mathrm{P}(20), \mathrm{P}(40), \mathrm{P}(60), \mathrm{P}(80)$, and $\mathrm{P}(100)$ at $\mathrm{p}=100 \mathrm{~Pa}$ and $\mathrm{T}=340 \mathrm{~K} . \mathrm{P}(20)$ of ${ }^{14} \mathrm{C}^{16} \mathrm{O}_{2}$, assuming ${ }^{14} \mathrm{C} /{ }^{12} \mathrm{C}=10^{-12}$, is shown as a reference.

O. This process was found to have a considerable effect on the observed OG signal. The RGA was used to measure the partial pressure of $\mathrm{CO}_{2}$ and $\mathrm{CO}$ after plasma ignition as shown in Figure $8 \mathrm{~b}$ and c for 2 different pressures. The $\mathrm{CO}_{2}$ amount was found to 1) drop immediately and steeply upon ignition of the plasma and 2) stabilize and subsequently decrease slowly over time. Moreover, oscillations in the $\mathrm{CO}_{2}$ and $\mathrm{CO}$ partial pressures over time were observed (Figure 8c). The latter effect was more pronounced at lower pressure as well as having a shorter onset time. It could be argued that the oscillations may stem from instabilities in the RF oscillator circuit, but the fact that the $\mathrm{CO}_{2}$ and $\mathrm{CO}$ oscillations were $180^{\circ}$ out of phase suggest that they are more likely driven by the kinetics of the chemical reactions in the plasma.

Concerning the oscillations, it is noteworthy that in spite of the known collision rates at these pressures, such "slow" oscillation in the reaction from $\mathrm{CO}_{2}$ to $\mathrm{CO}$ is taking place on periods of tens to hundreds of seconds depending on the base pressure. Oscillatory reactions between $\mathrm{CO}_{2}$ and $\mathrm{CO}$ are known to occur on surfaces with metal catalysts (Hendriksen et al. 2010). Such reaction oscillations have previously not been observed in the bulk gas phase, with or without a catalyst. This is suspected to be the first time $\mathrm{CO}_{2} \leftrightarrow \mathrm{CO}$ reaction oscillations are observed in a non-surface environment. This phenomenon is currently being investigated at our laboratory.

The resemblance between the oscillations seen in the RGA measurements (Figure 8c) and the instabilities in the OG signal (Figure 8a), in terms of waveform, onset time, and oscillation frequency, makes it fair to assume that both phenomena have the same origin, namely the plasma-induced oscillation in the concentration of $\mathrm{CO}_{2}$. Such chemical reaction oscillations necessitate the establishment of a parameter range where they do not occur or are stable over timescales corresponding to a measurement sequence.

Another issue that was noted was the fact that performing 2 consecutive measurements, with identical settings for the lasers, RF oscillators, etc., but with a repeated gas fill, could lead to variation of around $20 \%$ in the OG signal. This was probably due to the fact that the plasma parameters were sensitive to small pressure changes, and that it was difficult to obtain exactly the same pressure in 2 consecutive fills. It should be noted that these problems might be mitigated by measurements in dynamic, instead of static, mode. 

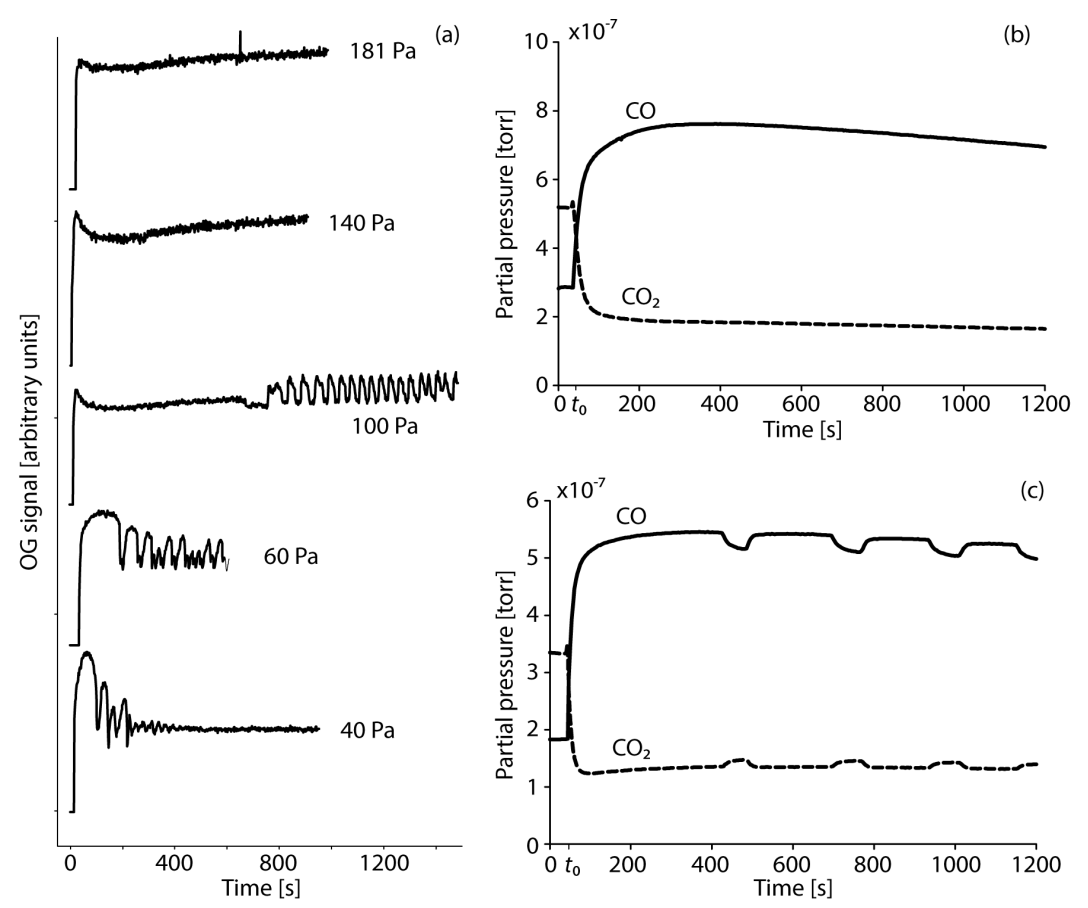

Figure 8 Oscillating intensity in the OG measurements (a) where the start, frequency, and duration of the oscillations were dependent on the pressure. The partial pressures of $\mathrm{CO}_{2}$ and $\mathrm{CO}$ as a function of time at pressures $\mathrm{p}=100 \mathrm{~Pa}(\mathrm{~b})$ and $50 \mathrm{~Pa}(\mathrm{c})$, measured with the RGA, are shown to the right. Here, time $t_{0}$ indicates plasma ignition.

\section{Pressure}

One of the parameters affecting the OG signal is the gas pressure. Based on the description given by Murnick et al. (2008) and Ilkmen (2009), the signal intensity should be proportional to the pressure. In our system, we have observed a rather limited pressure range in which ICOGS measurements can be performed, spanning from a few tens up to a few hundred Pa. At a RF frequency of $38 \mathrm{MHz}$, the operational range was 20-200 $\mathrm{Pa}$, whereas for pressures up to $500 \mathrm{~Pa}$, one needs to reduce the RF frequency to about $15 \mathrm{MHz}$. At lower pressures, the OG signal shows instability rendering it not useful, whereas at higher pressures the plasma could not be ignited with our oscillator. However, these limitations were set by the design of the oscillator circuit, and could probably, if required, be reduced by redesigning or changing the plasma source.

\section{Gas Composition}

There are a variety of gas compositions that could be investigated for studying $\mathrm{CO}_{2}$ samples, in analogy to the gas chemistry of the $\mathrm{CO}_{2}$ laser (Duarte 1995). Here, we have used 2 alternatives: 1) pure $\mathrm{CO}_{2}$ gas and 2) a mixture of $90-95 \% \mathrm{~N}_{2}$ and $5-10 \% \mathrm{CO}_{2}$. We are in the process of evaluating the 2 mixtures. The latter leads to an increase in the signal strength due to $\mathrm{N}_{2}$ molecules' collisional excitation of the $\mathrm{CO}_{2}$ molecules (one of the main principles behind $\mathrm{CO}_{2}$ lasers) while adding an additional absorption background from nitrogen. Pure $\mathrm{CO}_{2}$, on the other hand, lacks the added background but has a weaker signal. 


\section{G Eilers et al.}

\section{CONCLUSION}

The ICOGS setup in our laboratory is operational but not fully developed. Various aspects and operational details of the ICOGS have been studied and presented. A number of issues need to be addressed before performing analytical ${ }^{14} \mathrm{C}$ measurement of $\mathrm{CO}_{2}$. First, the absorption background, originating from non- ${ }^{14} \mathrm{CO}_{2}$ entities such as the ro-vibrational absorption bands of ${ }^{13} \mathrm{CO}_{2}$ and ${ }^{12} \mathrm{CO}_{2}$, as well as the pressure-dependent $\mathrm{N}_{2}$ background (for gas mixtures) have to be thoroughly characterized. Second, the signal stability and reproducibility have to be improved. This includes the oscillatory variations in the amount of $\mathrm{CO}_{2}$ in the gas cell $\left(\mathrm{CO}_{2} \leftrightarrow \mathrm{CO}\right)$. Third, calibration data should to be secured, using the in-house AMS facility. Work on addressing these issues is currently underway, with the aim of using ICOGS for measuring $\mu$ g-size sample in the 1-2 Modern range. We have so far been unable to detect ${ }^{14} \mathrm{C}$ unambiguously in the range of $0.2-10$ Modern.

\section{ACKNOWLEDGMENTS}

The authors wish to acknowledge the Swedish Research Council for supporting this project (Ref. A0442201). Dr Anders Holmberg, Karolinska Institute, Stockholm, is gratefully acknowledged for providing us with the ${ }^{14} \mathrm{C}$-labeled dextran samples. We thank Prof Daniel Murnick for his support throughout this project.

\section{REFERENCES}

Bradley LC, Soohoo KL, Freed C. 1986. Absolute frequencies of lasing transitions in nine $\mathrm{CO}_{2}$ isotopic species. IEEE Journal of Quantum Electronics 22(2): 234-67.

Chedin A. 1975. The carbon dioxide molecule: potential, spectroscopic, and molecular constants from its infrared spectrum. Journal of Molecular Spectroscopy 76(1-3):430-91

Deju N, Kan T, Wolga GJ. 1968. Gain distribution, population densities, and rotational temperature for the $\left(00^{0} 1\right)-\left(10^{0} 0\right)$ rotation-vibration transitions in a flowing $\mathrm{CO},-\mathrm{N},-\mathrm{He}$ laser. IEEE Journal of Quantum Electronics 4(5):256-60.

Demtröder W. 1981. Laser Spectroscopy. Berlin: Springer-Verlag.

Duarte FJ. 1995. Tunable Lasers Handbook. San Diego: Academic Press. 63 p.

Galli I, Bartalini S, Borri S, Cancio P, Mazzotti D, De Natale P, Giusfredi G. 2011. Molecular gas sensing below parts per trillion: radiocarbon-dioxide optical detection. Physical Review Letters 107:270802, doi: 10.1103/PhysRevLett.107.270802.

Hartmann B, Kleman B. 1966. Laser lines from $\mathrm{CO}_{2}$ in the 11 to 18 micron region. Canadian Journal of Physics 44:1609-12.

Hendriksen BLM, Ackermann MD, van Rijn R, Stoltz D, Popa I, Balmes O, Resta A, Wermeille D, Felici R, Ferrer S, Frenken JWM. 2010. The role of steps in surface catalysis and reaction oscillations. Nature Chemistry 2:730-4.
Huang X, Yung YL. 2004. A common misunderstanding about the Voigt line profile. Journal of the Atmospheric Sciences 61(13):1630-2.

Ilkmen E. 2009. Intracavity optogalvanic spectroscopy for radiocarbon analysis with attomole sensitivity [PhD thesis]. New Brunswick: Rutgers University.

Labrie D, Reid J. 1981. Radiocarbon dating by infrared laser spectroscopy. Applied Physics A 24(4):381-6.

Lappin G, Garner CR. 2003. Big physics, small doses: the use of AMS and PET in human microdosing of development drugs. Nature Reviews 2:233-40.

May RD, May PH. 1986. Solid-state radio frequency oscillator for optogalvanic spectroscopy: detection of nitric oxide using the 2-0 overtone transition. Review of Scientific Instruments 57(9):2242-5.

Meyer TW, Rhodes CK, Haus HA. 1993. High-resolution line broadening and collisional studies in $\mathrm{CO}_{2}$ using nonlinear spectroscopic techniques. Physical Review A 12(5): 1993-2008.

Murnick DE, Peer BJ. 1994. Laser-based analysis of carbon isotope ratios. Science 263(5149):945-7.

Murnick DE, Dogru O, Ilkmen E. 2008. Intracavity optogalvanic spectroscopy. An analytical technique for ${ }^{14} \mathrm{C}$ analysis with subattomole sensitivity. Analytical Chemistry 80(13):4820-4.

Salehpour M, Possnert G, Bryhni H. 2008. Subattomole sensitivity in biological accelerator mass spectrometry. Analytical Chemistry 80(10):3515-21.

Silfvast WT. 1996. Laser Fundamentals. Cambridge: Cambridge University Press. 